\title{
Sur un nouvel Amphipode souterrain de Bulgarie Niphargus kochianus melticensis $n$. ssp.
}

\author{
par \\ Dan DANCAU* et Stoitze ANDREEV**
}

Les Niphargides de Bulgarie sont, en général, connus grâce aux travaux publiés par L. Fage (1926), St.et G. Karaman (1959), et St. Andreev (1966). Jusqu'à présent on a mentionné ou décrit un petit nombre d'espèces et sous-espèces (7), la plupart étant des endémiques.

Pendant une excursion d'études que nous avons effectuée au mois d'Octobre 1970, dans la région de Loveč nous avons récolté dans un puits du village Sokolovo, de nombreux exemplaires de Niphargus kochianus, espèce qui n'avait pas été identifiée en Bulgarie. A notre avis il s'agit d'une nouvelle sous-espèce que nous nommerons Niphargus kochianus melticensis*** n. ssp.

Cette sous-espèce, dont la taille des adultes atteint seulement $3 \mathrm{~mm}$ est caractérisée par les particularités morphologiques suivantes: maxille I avec le lobe interne pourvu d'un seul poil et le lobe externe garni distalement de 7 épines masticatrices, dont 6 unidentées et la 7-ème, l'épine interne, pourvue de 5-6 petites dents; gnathopodes à carpes beaucoup plus longs que les propodes; dactyles de tous les péréiopodes avec des griffes très longues par rapport à leurs socles; péréiopodes V-VII ayant le lobe distal de l'angle postérieur proéminent et arrondi; telson, plus long que large divisé jusqu'à la base et portant sur chaque lobe, au milieu du bord externe, une épine et deux longues soies aristées.

Nous présentons ici la description de cette nouvelle sous-espèce.

\section{Niphargus kochianus melticensis $n$. ssp.}

Holotype: une femelle de $2,6 \mathrm{~mm}$, avec des oostégites bien développés et pourvus de longues soies sur leurs bords libres, récoltée le 3-XI-1970, dans un puits du village Sokolovo (district Loveč). Les 8 préparations microscopiques de l'holotype se trouvent dans la collection de l'Institut Zoologique et Musée de Sofia, enregistrées sous le numéro 15.

Paratypes: 50 exemplaires mâles, femelles et juvéniles récoltés dans le même puits que l'holotype, ainsi que dans 2 autres puits voisins (le 26-X et 3-X - 1970). Les

\footnotetext{
* Institut de Spéléogie „Emil Racovitza” 8 Rue D-r Capça, Bucarest, Rumanie.

** Institut et Musée de Zoologie, 1 Boulevard Ruski, Sofia, Bulgarie.

*** D'après le mot Melta, vieil nom romain de la ville Loveč
} 
paratypes se trouvent dans la même collection que l'holotype ainsi que dans la collection de l'Institut de Spéologie 'Emil G. Racovitza' de Bucarest (15 exemplaires).

\section{DESCRIPTION DE L'HOLOTYPE}

Antenne I dépassant la moitié du corps; les articles du pédoncule décroissent en longueur depuis la base; le flagelle principal composé de 17 articles. Les bâtonnets hyalins sont égaux ou un peu plus courts que les articles correspondants. Flagelle accessoire un peu plus long que les deux premiers articles du flagelle principal; l'article basal trois fois plus long que le suivant.

Antenne II à flagelle de 6 articles.

Mandibules (fig. 1, C-E): Le bord tranchant et la lame accessoire différents chez les deux mandibules. Entre le bord tranchant et le processus molaire, il y a une rangée de 7 épines. Le deuxième article du palpe a 3 soies au bord interne et le dernier article a 12 soies sur le bord interne, deux soies sur la face interne et une soie sur la face externe.

Maxilles I (fig. 1, B): lobe interne allongé et pourvu d'une seule soie distale. Lobe externe garni distalement de 7 épines masticatrices, dont 6 unidentées et la 7-ème, l'épine interne, pourvue de 5-6 petites dents. Palpe sur le second article ayant 4 soies distales, insérées sous-apicalement. Sur le même article du côté interne, il y a un poil et sur le bord externe quelques très fines soies.

Maxillipède (fig. 1, A): lobe interne un peu plus court que le premier article du palpe et portant 2 dents et une épine distalement et 3 épines sous-apicalement du côté interne. Lobe externe atteignant $2 / 3$ du deuxième article du palpe.

Gnathopodes I et II (fig. 2, A-D; fig. III, K-L): carpe beaucoup plus long que le propode, spécialement pour la deuxième paire de gnathopodes; les dactyles portant une seule soie. Chez les deux paires de gnathopodes, dans l'angle palmaire, il y a 2 épines, dont l'une plus courte. Le bord interne du propodite est pourvu de deux groupes de soies chez le premier gnathopode et de trois groupes chez le deuxième gnathopode. L'article ischial et méral des gnathopodes I et l'article ischial des gnathopodes II pourvus chacun d'une callosité à leurs bords sternaux.

Plaques coxales (fig. 2, A-B; fig. 3, A-E): I-III plus hautes que larges, IV a même largeur que hauteur, avec le bord postérieur concave.

Péréiopodes (fig. 3, C-J): de longueurs différentes $\left(\mathrm{P}_{3}: 1,20 \mathrm{~mm} ; \mathrm{P}_{4}: 1,90 \mathrm{~mm} ; \mathrm{P}_{5}\right.$ : $\left.1,44 \mathrm{~mm} ; \mathrm{P}_{6}: 1,98 \mathrm{~mm} ; \mathrm{P}_{7}: 2,01 \mathrm{~mm}\right)$.

L'article basal des trois derniers péréiopodes est plus long que large, avec le lobe distal de l'angle postérieur proéminent et arrondi. Les dactyles de tout les 

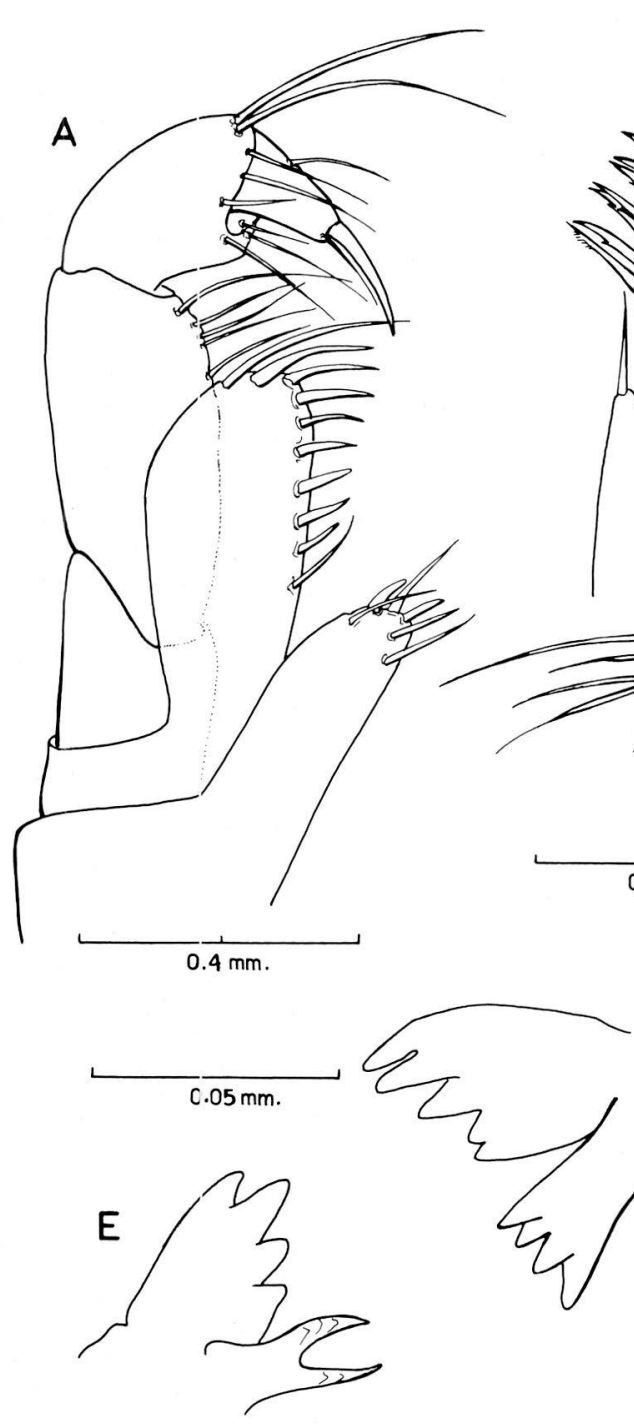

C

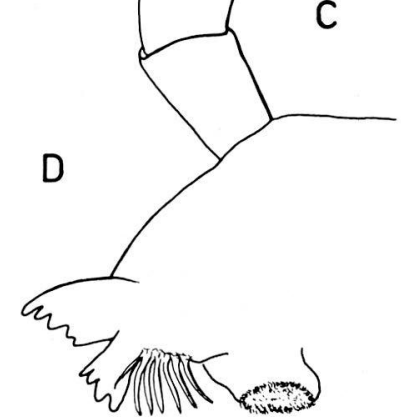

Fig. 1. A - H: Niphargus kochianus melticensis $\mathrm{n}$. ssp. Holotype. A - maxillipède; $\mathrm{B}$ - maxille 1; C - mandibule; D - E - bord tranchant et lame accessoire - droit et gauche. 


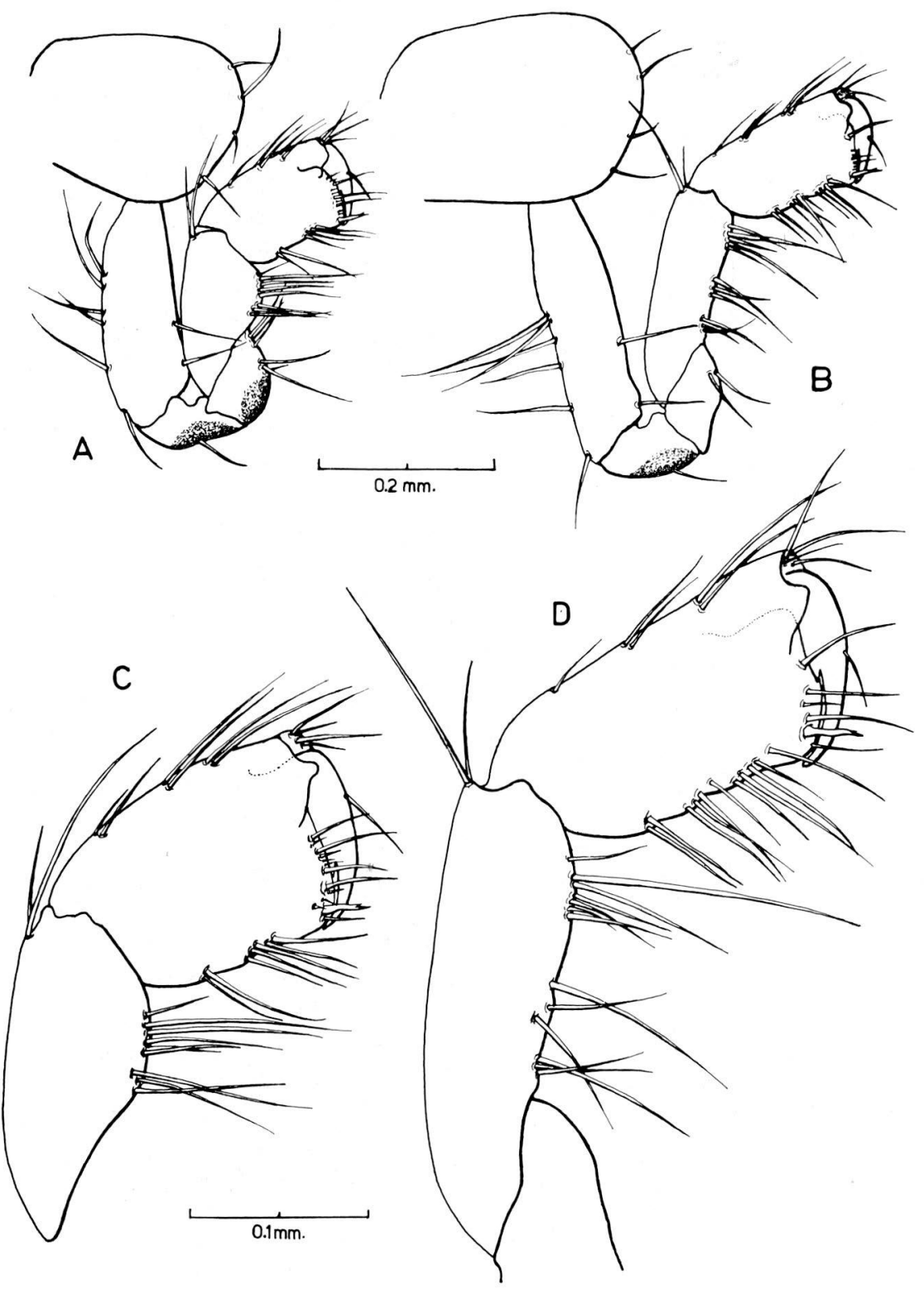

Fig. 2. A - D: Niphargus kochianus melticensis n. ssp. Holotype. A - gnathopode 1; B - gnathopode $2 ; \mathrm{C}$ - gnathopode $1 ; \mathrm{D}$ - gnathopode 2 . 

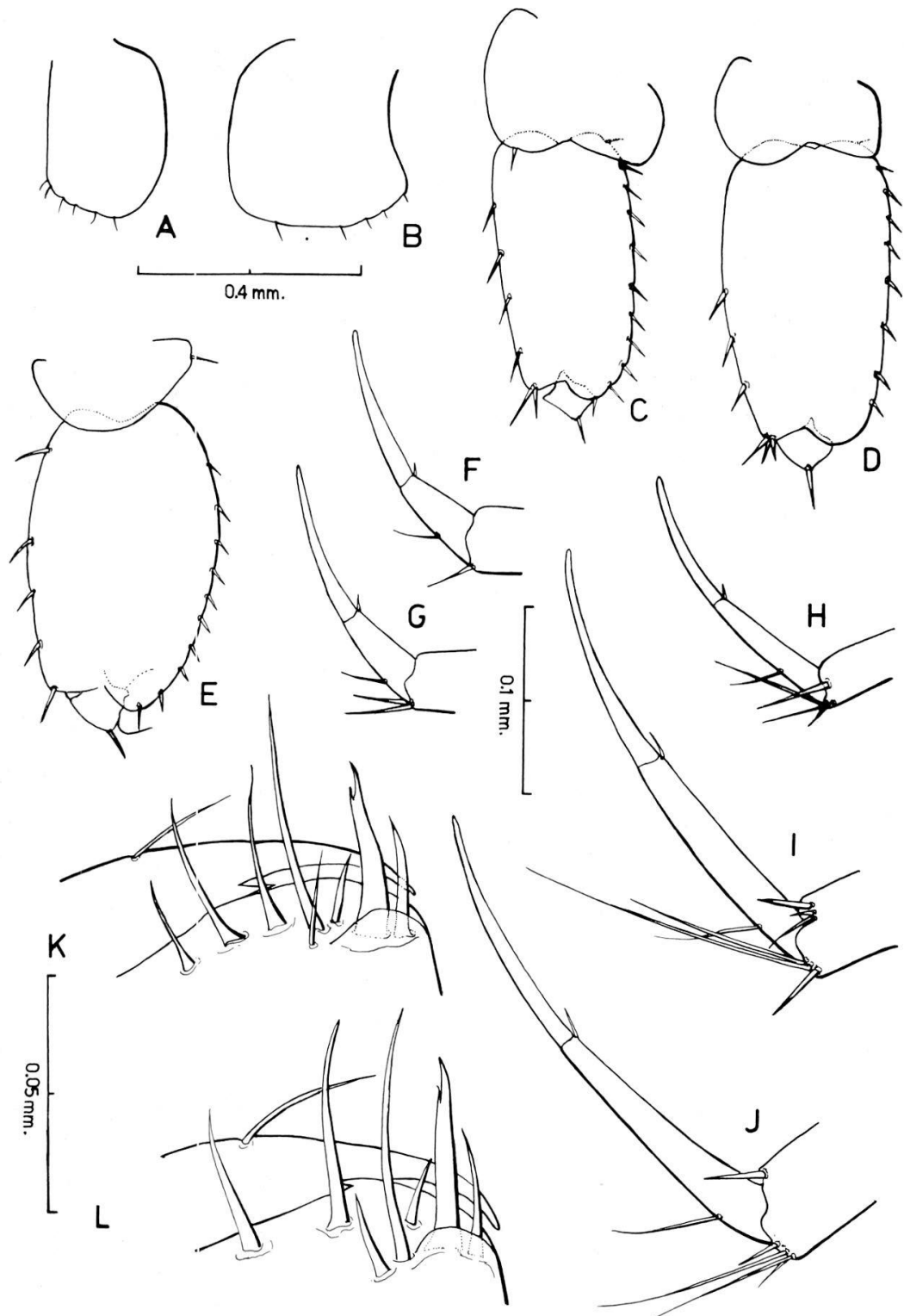

Fig. 3. A - L: Niphargus kochianus melticensis n. ssp. Holotype A, B, C, D et E - plaques coxales de III, IV, V, VI, et VII péréiopode; C, D et E - articles basales de V, VI et VII péréiopode $\mathrm{F}, \mathrm{G}, \mathrm{H}, \mathrm{I}, \mathrm{J}$ - dactyles des péréiopodes $\mathrm{V}$, VI et VII; $\mathrm{K}-\mathrm{L}$ - angles palmaires des gnathopodes 1,2 . 
péreìopodes ont des griffes très longues par rapport à leurs socles. Ainsi les griffes des dactyles de $\mathrm{P}_{3}$ et $\mathrm{P}_{4}$ sont 1,5 plus longues que leurs socles et chez les $\mathrm{P}_{5}-\mathrm{P}_{7}$, les griffes sont plus ou moins de la même longueur que leurs socles.

Les plaques épimérales (fig. 4, D): II et III ont leurs angles inféro-postérieurs aigus; les deux dernières sont armées d'une seule épine.

Pléopodes: avec 2 crochets par rétinacle.

Uropodes I et II (fig. 4, E, F): ayant des branches subégales, élancées.

Uropodes III (fig. 4, A): indifférenciés. Le pédoncule est deux fois plus long que la rame interne. Branche externe à premier article 4 fois plus long que le deuxième. Deuxième article de l'urosome armé de 2 épines subégales.

Telson (fig. 4, B, C): plus long que large, profondément échancré presque jusqu'à la

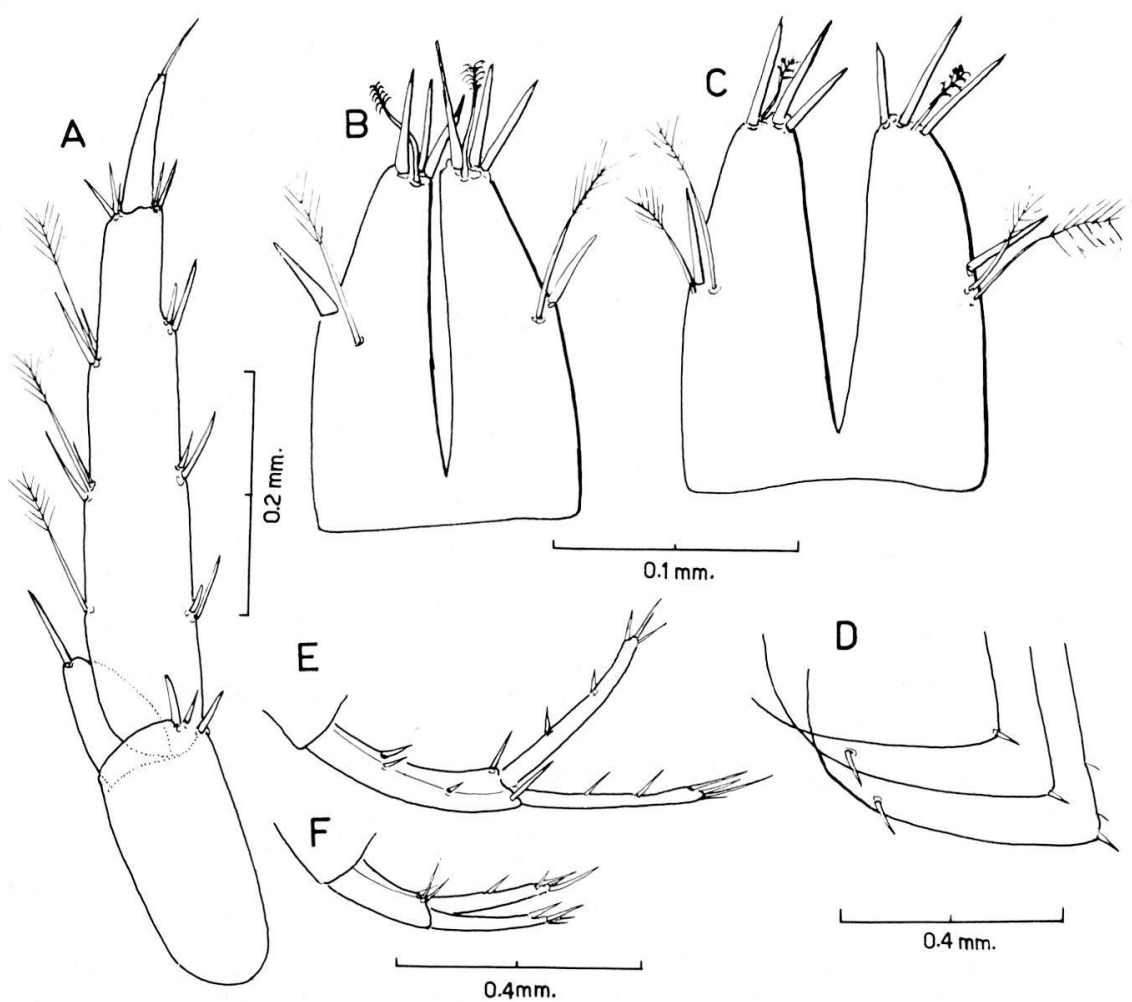

Fig. 4. A - F: Niphargus kochianus melticensis n. ssp. Holotype. A - uropode 1; B - telson; $\mathrm{C}$ - telson; $\mathrm{D}$ - plaques épimèrales; $\mathrm{E}$ - uropode $1 ; \mathrm{F}$ - uropode 2. 
base. Chaque lobe présentant trois épines distales et un petit poil aristé; au milieu des bords externes une épine et deux longues soies aristées; bords internes dépourvus d'épines.

\section{OBSERVATIONS SUR LES PARATYPES}

Les exemplaires examinés présentent, en général, les caractères morphologiques que nous avons mentionnés pour l'holotype. Nous n'avons pas observé des différences importantes en ce qui concerne les caractères morphologiques des exemplaires mâles et femelles de la même taille. Quelques petites différences quantitatives existent toutefois entre les exemplaires de taille différente, marquées par le nombre des articles pour les flagelles des deux antennes et par la chétotaxie des appendices.

Parmi les 50 paratypes (mâles, femelles et juvéniles) ayant une taille qui varie 1,1 à $3 \mathrm{~mm}$ nous avons identifié une femelle de $2,4 \mathrm{~mm}$ ayant un seul oeuf dans le marsupium.

\section{AFFINITÉS ET DIFFÉRENCES}

Par la structure de la maxille I qui a un seul poil sur l'apex du lobe interne et une seule épine pectinée parmi les 7 épines du lobe externe, notre sous-espèce se rapproche de $N$. kochianus tamanini Ruffo, petrosani Dobr. et Man., labacensis Sket, et minor Sket. De toutes celles-ci $N$. kochianus melticensis se distingue nettement par les péréiopodes ayant les griffes des dactyles très longues par rapport à leurs socles, ainsi que par la forme et la chétotaxie du telson.

\section{ÉCOLOGIE}

Les puits que nous avons prospectés dans le village Sokolovo ont une profondeur de 8 à $24 \mathrm{~m}$. et un diarnètre de 0,80 à $1 \mathrm{~m}$. Les récoltes ont été effectuées avec le filet phréatobiologique Cvetkov.

A part Niphargus kochianus melticensis n. ssp. nous avons trouvé dans ces puits les Amphipodes Niphargus ablaskiri gueorguievi St. et G. Karaman, mentionnés seulement des grottes, et Bogidiella sp. ainsi que des Isopodes Microparasellidae (Microcharon sp.).

\section{RÉSUMÉ}

La note présente la description d'une nouvelle sous-espèce, premier représentant du groupe de Niphargus kochianus en Bulgarie.

La nouvelle sous-espèce se rapproche de $N$. $k$. tamanini Ruffo, $N$. $k$. petrosani Dobr. et Manolache, $N$. $k$. labacensis Sket, $N$. $k$. minor Sket, par la maxille I ayant 
un seul poil sur le lobe interne et une seule épine pectinée du lobe externe. Mais elle diffère nettement de toutes les sous-espèces, par les griffes des dactyles très longues par rapport à leurs socles, ainsi que par la forme et la chétotaxie du telson.

\section{ZUSAMMENFASSUNG}

In dieser Mitteilung wird eine neue Unterart beschrieben, die als erster Vertreter der Gruppe Niphargus kochianus in Bulgarien auftritt.

Die neue Unterart steht $N$. $k$. tamanini Ruffo, $N$. $k$. petrosani Dobr., Man., $N . k$. labacensis Sket, $N$. $k$. minor Sket nahe nach Maxilla I und einem einzigen Börstchen auf dem inneren Lappen und einem einzigen Kammdorn auf dem äußeren Lappen. Sie unterschiedet sich aber deutlich von allen Unterarten nach ihren Daktillennägeln - sehr lang im Vergleich zu ihrer Basis sowie nach der Form und Chetotaxie des Telsons.

\section{BIBLIOGRAPHIE}

ANDREEV, S., 1966. Eine neue Niphargus-Art aus Bulgarien, Int. J. of Speleol., Vol. 2.

FAGE, L., 1926. Sur un Niphargus des eaux souterraines de Bulgarie, Bul. d. L. Soc. Scien. Cluj, T. III, 2.

KARAMAN, St. et G., 1959. Beitrag zur Kenntnis der Niphargiden Bulgariens. Acta Mus. Mac. Scien. Nat. T. VI, No 7.

RUFFO, S., 1937. Un nuovo Niphargus delle acque sotterranee del Veneto. Bol. Ist. Entom. R. Univ. Bologna, X.

RUFFO, S., 1953. Studi sui Crostacei Anfipodi XXXVIII. Nuovi Niphargus (Amphipoda Gammaridae) della Venezia Tridentina, Rev. del Mus. di Stor. Nat. della Venezia Tridentina, Ann. XXX, fasc. II.

SKET, B., 1956. Einige neue Formen der Malacostraca (Crust.) aus Jugoslawien, Bulletin Scientifique, T. 3, No 3.

SCHELLENBERG, A., 1932. Bemerkungen über subterrane Amphipoden Grossbritanniens. Zool. Anz., Bd. 99.

SCHELLENBERG, A., 1933. Höhlenflohkrebse des Adelsberger Grottensystems nebst Bemerkung über Niphargus kochianus, Mitt. über Höhlen- und Karstforsch.

SCHELLENBERG, A., 1933. Weitere deutsche und ausländische Niphargiden. Zool. Anz. Bd., 102.

SCHELLENBERG, A., 1936. Subterrane Amphipoden Badens, nebst einem neuen Niphargus aus Polen. Zool. Anz. Bd. 113. 